\title{
Zur Theorie des nichtlokalen Supraleiters im Magnetfeld
}

\author{
W. Schattke \\ Institut für Theoretische Physik und Sternwarte der Universität Kiel \\ (Z. Naturforsch. 23 a, 1822-1833 [1968] ; eingegangen am 9. August 1968)
}

\begin{abstract}
Starting from the BCS-Theory, an upper bound for the free energy is obtained by a combination of a variational method and perturbation theory. The variational equations obtained are non-local. The parameters of the perturbation calculation are the vector potential and the spatial variations of the order parameter, which have to be small. Boundary conditions are set for the case of diffuse reflection and pair-breaking at the surface. As an example, the superconducting plate is discussed.
\end{abstract}

\section{A. Problemstellung}

Eine große Zahl von Supraleitern zeigt unter einem statischen Magnetfeld ein Verhalten, das sich in einer gewissen Umgebung der Sprungtemperatur in guter Näherung mit der GinzBurg-Landau-Theorie $^{1,2}$ beschreiben läßt. Diese Supraleiter zeichnen sich auch bei tiefen Temperaturen durch eine im Verhältnis zur Kohärenzlänge $\xi_{0}\left(=\hbar v_{\mathrm{F}} / \pi \varepsilon_{0}\right)$ große Eindringtiefe aus, was mit Hilfe des Ginzburg-Landau-Parameters $\varkappa$ durch $x \gtrsim 1 / \sqrt{ } 2$ ausgedrückt wird. Wir beschränken uns auf den reinen Fall, d. h. auf Supraleiter, die keine Verunreinigungen enthalten.

Supraleiter, für die $x \ll 1 / \sqrt{ } 2$ gilt oder deren äußere Abmessungen klein gegen $\xi_{0}$ sind, verlangen eine Theorie, die die räumliche Korrelation der Elektronen eines Cooper-Paares berücksichtigt. Die nichtlokale Beziehung zwischen Vektorpotential und Strom, die Pippard ${ }^{3}$ angenommen hat, erfüllt diese Forderung insoweit als der Ordnungsparameter als konstant vorausgesetzt werden kann, so z. B. bei der Berechnung der Eindringtiefe eines schwachen Magnetfeldes. Aber abgesehen davon, daß der Ordnungsparameter auch vom Magnetfeld abhängen kann, fehlt dann die für den Meissner-Effekt notwendige positive Oberflächenenergie.

Supraleiter mit $\varkappa \geqq 1 / \sqrt{2}$, Supraleiter 2. Art, benehmen sich definitionsgemäß bezüglich des Magnetfeldes lokal, was sich in einer relativ großen Eindringtiefe äußert. Nichtsdestoweniger kann aber der Ordnungsparameter, abgesehen von der unmittelbaren Umgebung der Sprungtemperatur, stark

1 W. L. Ginzburg u. L. D. Landau, Zh. Eksperim. Teor. Fiz. 20, 1064 [1950] (s. z. B.: W. L. Ginzburg, Abh. sowjet. Phys., F. II, S. 135, Berlin 1951). - L. P. Gorkov, Soviet Phys.-JETP 9, 1364 [1959]. - L. Tewordt, Phys. Rev. 132, 595 [1963]. - N. R. Werthamer, Phys. Rev. 132, 663 [1963]. ortsabhängig sein. Damit wird die Anwendung lokaler Gleichungen wie der Ginzburg-Landau-Gleichungen fraglich, bzw. bedarf sie einer Rechtfertigung.

In dieser Arbeit werden Bestimmungsgleichungen für Vektorpotential und Ordnungsparameter aus der BCS-Theorie ${ }^{4}$ abgeleitet, die die gesuchten Größen in allgemeinen nichtlokalen Ausdrücken enthalten. Die Gleichungen verbinden die nichtlokale Pippardsche Theorie mit nichtlinearen Gleichungen des Ginzburg-Landau-Typs. Pippards Gleichung ergibt sich hieraus in der Näherung, daß der Ordnungsparameter konstant ist. Die Ginzburg-Landau-Gleichungen folgen, wenn man schwache Ortsabhängigkeit für Vektorpotential und Ordnungsparameter fordert, d. h. höhere Ordnungen in den räumlichen Ableitungen vernachlässigt.

Wir benötigen als Voraussetzung nur, daß die mittlere absolute Abweichung des Ordnungsparameters von seinem Ortsmittel und das Magnetfeld klein sein müssen. Die Größe des Ordnungsparameters braucht nicht eingeschränkt zu werden, und folglich gelten die Gleichungen für alle Temperaturen.

Formal erscheinen die nichtlokalen Beziehungen als Integrodifferentialgleichungen in den Ortskoordinaten. Solange man den massiven Supraleiter mit im Unendlichen liegender Oberfläche betrachtet, hängen die Integralkerne nur vom Abstand zweier Orte ab, und der Bereich, in dem die Integralkerne wesentlich von Null verschieden sind, ist durch $\xi_{0}{ }^{3}$ gegeben.

Die mikroskopische Ableitung geschieht für den unendlich ausgedehnten Supraleiter. Im Falle des endlichen Supraleiters werden diffuse Randbedin-

2 G. Eilenberger, Z. Phys. 182, 427 [1965].

3 A. B. Pippard, Proc. Roy. Soc. London A 216, 547 [1953].

4 J. Bardeen, L. N. Cooper u. J. R. Schrieffer, Phys. Rev. 108, 1175 [1957]. 
gungen für den Ordnungsparameter $\Delta(\boldsymbol{r})$ in ähnlicher Weise eingeführt, wie sie für das Vektorpotential $\boldsymbol{A}(\boldsymbol{r})$ üblich sind. $|\Delta(\boldsymbol{r})|$ bzw. grad $|\Delta(\boldsymbol{r})|$ werden, je nachdem ob wir an der Oberfläche Paarbrechung bzw. diffuse Paarreflexion verlangen, außerhalb des Supraleiters gleich Null gesetzt. Bezüglich des Magnetfeldes erscheint es nur sinnvoll, außerhalb das Verschwinden von $\boldsymbol{A}(\boldsymbol{r})$ zu fordern.

Den Ausgangspunkt der mikroskopischen Ableitung bildet der BCS-Hamilton-Operator. In der Wechselwirkung sind allerdings auch die Elektronenpaare enthalten, deren Gesamtimpuls nicht verschwindet. Die Wechselwirkung ist im Impulsraum durch ein Kastenpotential gegeben, was sich im Ortsraum formal durch den Gorkov-Ansatz ${ }^{5}$ beschreiben läßt. Das Magnetfeld geht nur über die kinetische Energie in den Hamilton-Operator ein. Wie im translationsinvarianten Fall werden je zwei Einteilchenoperatoren in der Wechselwirkung gemäß einer verallgemeinerten Hartree-Fock-Approximation durch eine $c$-Zahl ersetzt, deren Wert in selbstkonsistenter Weise bestimmt wird. Im Ortsraum ist diese $c$-Zahl der Ordnungsparameter, der die ortsunabhängige Energielücke ersetzt. Um einen Näherungswert für $\Delta(\boldsymbol{r})$ zu finden, benützen wir das Bogoliubovsche Variationsverfahren. Da die Eigenwerte des ErsatzHamilton-Operators, der alle in den Erzeugungsbzw. Vernichtungsoperatoren bilinearen Anteile enthält, nicht bekannt sind, wird dem Variationsverfahren eine Störungsrechnung überlagert. Die Abweichung des Ordnungsparameters von seinem räumlichen Mittelwert und das Vektorpotential werden als kleine Größen behandelt und stellen die Entwicklungsparameter dar. Die Entwicklung wird nach der zweiten Ordnung abgebrochen. Man erhält so eine obere Schranke für das thermodynamische Potential, die gemäß dem Bogoliubovschen Variationsverfahren bezüglich $\Delta(\boldsymbol{r})$ minimalisiert wird. Die Variationsgleichung und die Maxwellschen Gleichungen bilden das Gleichungssystem zur Bestimmung von $\Delta(\boldsymbol{r})$ und $\boldsymbol{A}(\boldsymbol{r})$.

Das Beispiel der supraleitenden Platte wird gerechnet, um die Randbedingungen zu untersuchen. Dabei werden Näherungen für die Integralkerne benutzt, die einerseits die wichtigsten Merkmale der exakten Kerne zeigen, andererseits aber noch leicht zu handhaben sind.

\footnotetext{
5 L. P. Gorkov, Soviet Phys.-JETP 7, 505 [1958].
}

\section{B. Obere Schranke der Freien Energie}

Wir benutzen den Hamilton-Operator der BCSTheorie in der von Gonkov ${ }^{5}$ angegebenen Form

$$
\begin{aligned}
& H=\frac{1}{2 m} \sum_{\alpha} \int \mathrm{d}^{3} \boldsymbol{r} \psi_{\alpha}^{+}(\boldsymbol{r})\left(\frac{\hbar}{i} \nabla-\frac{e}{c} \boldsymbol{A}\right)^{2} \psi_{\alpha}(\boldsymbol{r}) \\
& +\int \mathrm{d}^{3} \boldsymbol{r} \mathrm{d}^{3} \boldsymbol{r}^{\prime} V\left(\boldsymbol{r}-\boldsymbol{r}^{\prime}\right) \psi_{\uparrow}^{+}(\boldsymbol{r}) \psi_{\downarrow}^{+}\left(\boldsymbol{r}^{\prime}\right) \psi_{\downarrow}\left(\boldsymbol{r}^{\prime}\right) \psi_{\uparrow}(\boldsymbol{r}), \\
& V\left(\boldsymbol{r}-\boldsymbol{r}^{\prime}\right)=-V_{0} \delta\left(\boldsymbol{r}-\boldsymbol{r}^{\prime}\right),
\end{aligned}
$$

$\psi_{a}^{+}(\boldsymbol{r}), \psi_{a^{\prime}}\left(\boldsymbol{r}^{\prime}\right)$ sind Erzeugungs- bzw. Vernichtungsoperatoren des Ortsraumes mit den Vertauschungsregeln

$$
\left\{\psi_{a}(\boldsymbol{r}), \psi_{\alpha^{\prime}}^{+}\left(\boldsymbol{r}^{\prime}\right)\right\}=\delta_{\alpha, \alpha^{\prime}} \delta\left(\boldsymbol{r}-\boldsymbol{r}^{\prime}\right) .
$$

Der untere Index bezieht sich auf die $z$-Komponente des Spins. Die Wechselwirkung enthält implizit die Abschneidebedingung, d. h. nur Teilchen, deren Energien $\varepsilon_{\boldsymbol{k}}\left(=\hbar^{2} \boldsymbol{k}^{2} / 2 m-\zeta\right)$ innerhalb der Schale $\left|\varepsilon_{\boldsymbol{k}}\right|<\hbar \omega$ liegen, können in Wechselwirkung treten. $(e, m, \hbar \boldsymbol{k}, \hbar, \zeta, \omega, c$ bedeuten Elektronenladung, -masse, -impuls, Plancksches Wirkungsquantum, Fermi-Energie, Debye-Frequenz und Lichtgeschwindigkeit.)

Da wir den Fall schwacher Kopplung behandeln wollen, werden wir die Abschneidebedingung nur dann berücksichtigen, wenn eine Energieintegration divergiert. Der dabei auftretende Fehler geht mit der Größenordnung $\varepsilon_{\mathrm{T}} / \hbar \omega\left(\varepsilon_{\mathrm{T}}\right.$ BCS-Energielücke $)$ und ist vernachlässigbar.

Ebenso gilt für schwache Kopplung, daß sich der Hamilton-Operator unter der Eichtransformation

$$
\begin{gathered}
\psi_{a} \rightarrow \psi_{a} e^{-i \chi} \\
\boldsymbol{A} \rightarrow \boldsymbol{A}-(\hbar c / e) \nabla \chi
\end{gathered}
$$

nicht ändert.

Befindet sich das Elektronengas im Kontakt mit einem Teilchen- und Wärmereservoir, so läßt sich die Freie Energie mit Hilfe von (1) und des Teilchenzahloperators

$$
N=\sum_{\alpha} \int \mathrm{d}^{3} \boldsymbol{r} \psi_{\alpha}^{+}(\boldsymbol{r}) \psi_{\alpha}(\boldsymbol{r})
$$

folgendermaßen formulieren

$$
F_{\mathrm{s}}(\boldsymbol{A})=-k T \log \operatorname{Spur} e^{-\beta(H-\zeta N)}
$$

( $\beta=1 / k T, T$ abs. Temperatur, $k$ Boltzmann-Konstante). Der Strom ist dann durch

$$
\frac{\delta}{\delta \boldsymbol{A}} F_{\mathrm{s}}(\boldsymbol{A})=-\frac{1}{c} \boldsymbol{J}
$$


definiert. Um eine obere Schranke für $F_{\mathrm{s}}(\boldsymbol{A})$ zu bekommen, wird das Bogoliubovsche Variationsverfahren benutzt. Man spaltet vom Exponenten in Gl. (7) einen hermiteschen Anteil $H_{0}$ ab, der $(H-\zeta N)$ möglichst gut approximiert. Mit

$$
\begin{gathered}
H_{1}=H-\zeta N-H_{0}, \\
\left\langle H_{1}\right\rangle_{0}=\left(\operatorname{Spur} e^{-\beta H_{0}}\right)^{-1} \operatorname{Spur}\left(e^{-\beta H_{0}} H_{1}\right)
\end{gathered}
$$

gilt

$$
\boldsymbol{F}_{\mathrm{s}}(\boldsymbol{A}) \leqq-k T \log \operatorname{Spur} e^{-\beta H_{0}}+\left\langle H_{1}\right\rangle_{\mathbf{0}} .
$$

Durch Minimalisieren der rechten Seite von Gl. (11) nach den freien Parametern von $H_{0}$ erhält man die für diesen Ansatz tiefste obere Schranke. Mit dem Ordnungsparameter $\Delta(\boldsymbol{r})$ als Variationsparameter macht man für $H_{0}$ einen Ansatz, der bilinear in den Erzeugungs- und Vernichtungsoperatoren ist:

$$
\begin{aligned}
H_{0} & =\frac{1}{2 m} \sum_{\alpha} \int \mathrm{d}^{3} \boldsymbol{r} \psi_{\alpha}^{+}\left[\left(\frac{\hbar}{i} \nabla-\frac{e}{c} \boldsymbol{A}\right)^{2}-2 m \zeta\right] \psi_{a} \\
& -\int \mathrm{d}^{3} \boldsymbol{r}\left[\Delta(\boldsymbol{r}) \psi_{\uparrow}^{+} \psi_{\downarrow}^{+}+\text {h. c. }\right]+\frac{1}{V_{0}} \int \mathrm{d}^{3} \boldsymbol{r}|\Delta(\boldsymbol{r})|^{2} .
\end{aligned}
$$

Für $\boldsymbol{A}=0$ und konstantes $\Delta(\boldsymbol{r})=\varepsilon_{\mathrm{T}}$ liefert Gl. (12) die übliche ortsunabhängige BCS-Theorie. Letztere stellt den ungestörten Zustand einer anschließenden Störungsrechnung dar, die für die Auswertung der Spur in Gl. (11) gebraucht wird.
Der zweite Summand in Gl. (11) wird fortgelassen, weil bei Vernachlässigung der Hartree-Terme von $\left\langle H_{1}\right\rangle_{0}$ im absoluten Minimum ${ }^{2}$

$$
\left\langle H_{1}\right\rangle_{0}=0
$$

ist und außerdem wegen

$$
H_{1}=-V_{0} \int \mathrm{d}^{3} \boldsymbol{r}\left(\psi_{\uparrow} \psi_{\downarrow}-\frac{1}{V_{0}} \Delta^{*}(\boldsymbol{r})\right)\left(\psi_{\downarrow} \psi_{\uparrow}-\frac{1}{V_{0}} \Delta(\boldsymbol{r})\right)
$$

$$
\left\langle H_{1}\right\rangle_{0} \leqq 0
$$

gilt. Für die Störungsrechnung wird $H_{0}$ aufgeteilt

$$
H_{0}=H_{0}{ }^{0}+H_{A}+H_{\Delta},
$$

$H_{0}^{0}=\frac{1}{2 m} \sum_{\alpha} \int \mathrm{d}^{3} \boldsymbol{r} \psi_{\alpha}^{+}\left(-\hbar^{2} \nabla^{2}-2 m \zeta\right) \psi_{\alpha}$

$$
-\int \mathrm{d}^{3} \boldsymbol{r}\left(\Delta_{0} \psi_{\uparrow}^{+} \psi_{\downarrow}^{+}+\text {h. c. }\right)+\frac{1}{V_{0}} \int \mathrm{d}^{3} \boldsymbol{r}|\Delta(\boldsymbol{r})|^{2},
$$

$$
\begin{aligned}
H_{\boldsymbol{A}}=-\frac{\boldsymbol{e}}{2 m c} \sum_{\alpha} \int_{\alpha} \mathrm{d}^{3} \boldsymbol{r} \psi_{\alpha}^{+}\left[\frac{\hbar}{i}(\boldsymbol{A} \nabla)\right. \\
\left.+\frac{\hbar}{i}(\nabla \boldsymbol{A})-\frac{\boldsymbol{e}}{c} \boldsymbol{A}^{2}\right] \psi_{\alpha},
\end{aligned}
$$

$$
H_{\Delta}=-\int \mathrm{d}^{3} \boldsymbol{r}\left[\left(\Delta(\boldsymbol{r})-\Delta_{\mathbf{0}}\right) \psi_{\uparrow}^{+} \psi_{\downarrow}^{+}+\text {h. c. }\right] \text {. }
$$

Die Spur des statistischen Operators wird nach Schwinger entwickelt

$$
\begin{aligned}
& \operatorname{Spur} e^{-\beta H_{0}}=\left(\operatorname{Spur} e^{-\beta H_{0}^{0}}\right)\left[1-\int_{0}^{\beta} \mathrm{d} \beta^{\prime}\left\langle H_{A}\left(\beta^{\prime}\right)+H_{\Delta}\left(\beta^{\prime}\right)\right\rangle_{0,0}\right. \\
& \left.+\frac{1}{2} \int_{0}^{\beta} \mathrm{d} \beta^{\prime} \mathrm{d} \beta^{\prime \prime}\left\langle T\left(H_{\boldsymbol{A}}\left(\beta^{\prime}\right)+H_{\Delta}\left(\beta^{\prime}\right)\right)\left(H_{\boldsymbol{A}}\left(\beta^{\prime \prime}\right)+H_{\Delta}\left(\beta^{\prime \prime}\right)\right)\right\rangle_{0,0}+\ldots\right], \\
& \left\langle H_{\boldsymbol{A}}\right\rangle_{0,0} \equiv\left(\operatorname{Spur} e^{-\beta H_{0}{ }^{0}}\right)^{-1} \operatorname{Spur}\left(e^{-\beta H_{0}{ }^{0} H_{\boldsymbol{A}}}\right), \quad H_{\boldsymbol{A}}\left(\beta^{\prime}\right) \equiv e^{\beta^{\prime} H_{0}{ }^{0}} H_{A} e^{-\beta^{\prime} H_{0}{ }^{0}} .
\end{aligned}
$$

Der Zeitordnungsoperator $T$ ordnet hier nach der Größe von $\beta$. Es bleibt also folgende Minimalisierungsaufgabe

$$
\begin{gathered}
F_{\mathrm{s}}(\boldsymbol{A}) \leqq M(\Delta, \boldsymbol{A}) \equiv-k T \log \operatorname{Spur} e^{-\beta H_{0}{ }^{0}}+\frac{1}{\beta}\left[\int_{0}^{\beta} \mathrm{d} \beta^{\prime}\left\langle H_{\boldsymbol{A}}\left(\beta^{\prime}\right)+H_{\Delta}\left(\beta^{\prime}\right)\right\rangle_{0,0}\right. \\
\left.-\frac{1}{2} \int_{0}^{\beta} \mathrm{d} \beta^{\prime} \mathrm{d} \beta^{\prime \prime}\left\langle T\left(H_{\boldsymbol{A}}\left(\beta^{\prime}\right)+H_{\Delta}\left(\beta^{\prime}\right)\right)\left(H_{\boldsymbol{A}}\left(\beta^{\prime \prime}\right)+H_{\Delta}\left(\beta^{\prime \prime}\right)\right)\right\rangle_{0,0}^{\text {con }}+\ldots\right], \\
\frac{\delta}{\delta \Delta(\boldsymbol{r})} M(\Delta, \boldsymbol{A})=0, \quad \frac{\delta}{\delta \Delta^{*}(\boldsymbol{r})} M(\Delta, \boldsymbol{A})=0 .
\end{gathered}
$$

In Gl. (21) sind, durch den Index con(nected) angedeutet, nur die verbundenen Graphen zu berücksichtigen. Für die auftretenden Erwartungswerte gilt in der Grenze sehr großen Volumens das Wicksche Theo- 
rem in der speziellen Form

$$
\begin{aligned}
& \left\langle T \psi_{\alpha_{1}}^{+}(1) \psi_{\alpha_{2}}^{+}(2) \psi_{\alpha_{3}}(3) \psi_{\alpha_{4}}(4)\right\rangle_{0,0} \\
& \quad=\left\langle T \psi_{\alpha_{1}}^{+}(1) \psi_{\alpha_{4}}(4)\right\rangle_{0,0}\left\langle T \psi_{\alpha_{2}}^{+}(2) \psi_{\alpha_{3}}(3)\right\rangle_{0,0} \\
& \quad-\left\langle T \psi_{\alpha_{1}}^{+}(1) \psi_{\alpha_{3}}(3)\right\rangle_{0,0}\left\langle T \psi_{\alpha_{2}}^{+}(2) \psi_{\alpha_{4}}(4)\right\rangle_{0,0}+\left\langle T \psi_{\alpha_{1}}^{+}(1) \psi_{\alpha_{2}}^{+}(2)\right\rangle_{0,0}\left\langle T \psi_{\alpha_{3}}(3) \psi_{\alpha_{4}}(4)\right\rangle_{0,0}\left(l=\left(\boldsymbol{r}_{l}, \beta_{l}\right)\right)
\end{aligned}
$$

und entsprechend bei Produkten mit mehr Faktoren. Mit Hilfe von Gl. (23) lassen sich sämtliche Erwartungswerte auf Produkte von Greenschen Funktionen zurückführen:

$G_{12} \equiv\left\langle T \psi_{a}(1) \psi_{\alpha}^{+}(2)\right\rangle_{0,0}, \quad F_{12} \equiv\left\langle T \psi_{\downarrow}(1) \psi_{\uparrow}(2)\right\rangle_{0,0}=-\left\langle T \psi_{\uparrow}(1) \psi_{\downarrow}(2)\right\rangle_{0,0}$,

$F_{12}^{+} \equiv\left\langle T \psi_{\uparrow}^{+}(1) \psi_{\downarrow}^{+}(2)\right\rangle_{0,0}=-\left\langle T \psi_{\downarrow}^{+}(1) \psi_{\uparrow}^{+}(2)\right\rangle_{0,0}$.

Daraus ergibt sich

$$
\begin{aligned}
& M(\Delta, \boldsymbol{A})=-\frac{k T}{\Omega} \int \mathrm{d}^{3} \boldsymbol{r} \log \operatorname{Spur} e^{-\beta H_{0^{0}}}-\frac{e^{2}\langle N\rangle_{0,0}}{2 m c^{2} \Omega} \int \mathrm{d}^{3} \boldsymbol{r} \boldsymbol{A}^{2} \\
& -\frac{e^{2}}{4 m^{2} c^{2} \beta} \int \mathrm{d} 1 \mathrm{~d} l^{\prime} \mathrm{d} 2 \mathrm{~d} 2^{\prime} \delta\left(1-1^{\prime}\right) \delta\left(2-2^{\prime}\right) L(1) L(2)\left[F_{1^{\prime} 2^{\prime}}^{+} F_{12}-G_{21^{\prime}} G_{12^{\prime}}\right] \\
& -\frac{e}{2 m c \beta} \int \mathrm{d} \mathrm{d} \mathrm{d}^{\prime} \mathrm{d} 2 \delta\left(1-\mathrm{l}^{\prime}\right) L(1)\left[\left(\Delta\left(\boldsymbol{r}_{2}\right)-\Delta_{0}\right)\left(F_{1^{\prime} 2}^{+}+F_{21^{\prime}}^{+}\right) G_{12}+\left(\Delta^{*}\left(\boldsymbol{r}_{2}\right)-\Delta_{0}^{*}\right)\left(F_{12}+F_{21}\right) G_{21^{\prime}}\right] \\
& +\frac{1}{2 \beta} \int \mathrm{d} \mathrm{d} 2\left[\left(\Delta\left(\boldsymbol{r}_{1}\right)-\Delta_{0}\right)\left(\Delta\left(\boldsymbol{r}_{2}\right)-\Delta_{0}\right)\left(F_{12}^{+}\right)^{2}-2\left(\Delta\left(\boldsymbol{r}_{1}\right)-\Delta_{0}\right)\left(\Delta^{*}\left(\boldsymbol{r}_{2}\right)-\Delta_{0}^{*}\right)\left(G_{12}\right)^{2}\right. \\
& \left.\quad+\left(\Delta^{*}\left(\boldsymbol{r}_{1}\right)-\Delta_{0}^{*}\right)\left(\Delta^{*}\left(\boldsymbol{r}_{2}\right)-\Delta_{0}^{*}\right)\left(F_{12}\right)^{2}\right] \\
& \left(\int \mathrm{d} 1 \equiv \int \mathrm{d}^{3} \boldsymbol{r}_{1} \int_{0}^{\beta} \mathrm{d} \beta_{1}\right) \\
& L \quad L(1) \equiv \frac{\hbar}{i}\left(\boldsymbol{A}\left(\boldsymbol{r}_{1}\right) \nabla_{1}\right)+\frac{\hbar}{i}\left(\nabla_{1} \boldsymbol{A}\left(\boldsymbol{r}_{1}\right)\right)-\frac{e}{c}\left(\boldsymbol{A}\left(\boldsymbol{r}_{1}\right)\right)^{2}
\end{aligned}
$$

Für die nun folgenden, etwas mühseligen Rechnungen sei auf den Anhang verwiesen.

Die Differenz zwischen der Freien Energie des Supraleiters und der des Normalleiters lautet [Anhang Gl. (A 21)]

$$
\begin{aligned}
M(\Delta, \boldsymbol{A}) & -M(0, \boldsymbol{A})=\frac{1}{V_{0}} \int \mathrm{d}^{3} \boldsymbol{r}|\Delta(\boldsymbol{r})|^{2}+\int \mathrm{d}^{3} \boldsymbol{r} m\left(\left|\Delta_{0}\right|^{2}\right)-\left[\frac{1}{2 \Omega} \underset{|\varepsilon|<\hbar \omega}{\sum_{\boldsymbol{k}}} \frac{1}{E} \tanh \frac{\beta}{2} E\right] \int \mathrm{d}^{3} \boldsymbol{r}\left|\Delta(\boldsymbol{r})-\Delta_{0}\right|^{2} \\
& +\left[\frac{1}{8 \Omega} \sum_{\boldsymbol{k}}\left(\frac{1}{E^{3}} \tanh \frac{\beta}{2} E-\frac{\beta}{2 E^{2}}\left(\cosh \frac{\beta}{2} E\right)-2\right)\right] \int \mathrm{d}^{3} \boldsymbol{r}\left(2 \operatorname{Re}\left[\Delta_{0}^{*}\left(\Delta(\boldsymbol{r})-\Delta_{0}\right)\right]\right)^{2} \\
& +2 \int \mathrm{d}^{3} \boldsymbol{r} \mathrm{d}^{3} \boldsymbol{r}^{\prime} \operatorname{Re}\left[\left(\Delta(\boldsymbol{r})-\Delta_{0}\right) \Delta_{0}^{*}\right] K_{3}\left(\left|\boldsymbol{r}-\boldsymbol{r}^{\prime}\right|\right) \operatorname{Re}\left[\left(\Delta\left(\boldsymbol{r}^{\prime}\right)-\Delta_{0}\right) \Delta_{0}^{*}\right] \\
& +\frac{1}{2 m} \int \mathrm{d}^{3} \boldsymbol{r} \mathrm{d}^{3} \boldsymbol{r}^{\prime}\left(\frac{\hbar}{i} \operatorname{grad}_{l} \Delta(\boldsymbol{r})-\frac{2 e}{c} \Delta_{0} \boldsymbol{A}_{l}(\boldsymbol{r})\right) K_{1}\left(\left|\boldsymbol{r}-\boldsymbol{r}^{\prime}\right|\right)\left(-\frac{\hbar}{i} \operatorname{grad}_{l}^{\prime} \Delta^{*}\left(\boldsymbol{r}^{\prime}\right)-\frac{2 e}{c} \Delta_{0}^{*} \boldsymbol{A}_{l}\left(\boldsymbol{r}^{\prime}\right)\right) \\
& -\frac{2 e^{2}}{m c^{2}} \int \mathrm{d}^{3} \boldsymbol{r} \mathrm{d}^{3} \boldsymbol{r}^{\prime} \operatorname{rot}_{l} \boldsymbol{A}(\boldsymbol{r}) K_{2}\left(\left|\boldsymbol{r}-\boldsymbol{r}^{\prime}\right|\right) \operatorname{rot}_{l}^{\prime} \boldsymbol{A}\left(\boldsymbol{r}^{\prime}\right) \quad\left(E=\left(\varepsilon^{2}+\left|\Delta_{0}\right|^{2}\right)^{1 / 2}\right)
\end{aligned}
$$

wobei für $\Delta(\boldsymbol{r})$ der Wert einzusetzen ist, der das Funktional zum Minimum macht. Die Ableitung gilt für großes Normierungsvolumen $\Omega$ unter periodischen Randbedingungen; die $\boldsymbol{k}$-Summationen können durch Integrationen über ein kontinuierliches Impulsspektrum ersetzt werden. Die Integralkerne sind durch

und

$$
K_{n}\left(\left|\boldsymbol{r}-\boldsymbol{r}^{\prime}\right|\right) \equiv \frac{1}{\Omega} \sum_{\boldsymbol{q}} K_{n}(\boldsymbol{q}) \exp \left\{-i \boldsymbol{q}\left(\boldsymbol{r}-\boldsymbol{r}^{\prime}\right)\right\}
$$

$$
K_{1}(\boldsymbol{q})=-\frac{3 n}{2\left(\pi q \xi_{0} \varepsilon_{0}\right)^{2}} \int_{0}^{\infty} \frac{\mathrm{d} x}{X} \tanh (\alpha X)\left(x \log \left|\frac{x+1}{x-1}\right|-2\right)
$$




$$
\begin{gathered}
K_{2}(\boldsymbol{q})=\frac{3\left|\Delta_{0}\right|^{2}}{2 q^{2}} K_{1}(\boldsymbol{q})+\frac{3 n x_{0}^{2}}{32 q^{2}}\left[-\frac{\pi^{2}}{x_{0}} \tanh \left(\alpha x_{0}\right)+2 \int_{0}^{\infty} \frac{\mathrm{d} x}{x X} \tanh (\alpha X) \log \left|\frac{x+1}{x-1}\right|\right], \\
K_{3}(\boldsymbol{q})=-\frac{3 n}{8 \zeta\left|\Delta_{0}\right|^{2}}\left[\frac{\Lambda_{0}}{\Lambda}-\frac{\pi^{2}}{4} x_{0} \tanh \left(\alpha x_{0}\right)+\frac{1}{2} x_{0}^{2} \int_{0}^{\infty} \frac{\mathrm{d} x}{x X} \tanh (\alpha X) \log \left|\frac{x+1}{x-1}\right|\right]
\end{gathered}
$$

mit

$$
\begin{aligned}
X \equiv\left(x^{2}+x_{0}{ }^{2}\right)^{1 / 2}, \quad x_{0} \equiv \frac{2\left|\Delta_{0}\right|}{\pi q \xi_{0} \varepsilon_{0}}, \quad \alpha \equiv \frac{\beta \pi q \xi_{0} \varepsilon_{0}}{4}, \frac{A_{0}}{\Lambda} \equiv 1-\frac{\beta}{2} \int_{0}^{\infty} \mathrm{d} \varepsilon\left(\cosh \frac{\beta}{2} E\right)^{-2}, \\
n \equiv k_{\mathrm{F}}{ }^{3} / 3 \pi^{2}, \quad q \equiv|\boldsymbol{q}|
\end{aligned}
$$

gegeben. Der zweite, dritte und vierte Summand in Gl. (27) lassen sich im Rahmen unserer Näherung als Glieder einer Taylor-Entwicklung von $m\left(|\Delta(\boldsymbol{r})|^{2}\right)$ um $\left|\Delta_{0}\right|^{2}$ auffassen und deswegen durch

$$
\begin{gathered}
\int \mathrm{d}^{3} \boldsymbol{r}\left[m\left(|\Delta(\boldsymbol{r})|^{2}\right)+m^{\prime}\right]=\int \mathrm{d}^{3} \boldsymbol{r}\left[\frac{1}{\Omega} \sum_{\boldsymbol{k}}\left(\varepsilon-\tilde{E}-2 k T \log \frac{1+\exp \{-\beta \tilde{E}\}}{1+\exp \{-\beta \varepsilon\}}\right)+m^{\prime}\right] \\
m^{\prime} \equiv\left[\frac{1}{2 \Omega} \sum_{\substack{k \\
|\varepsilon|<\hbar \omega}} \frac{1}{E} \tanh \frac{\beta}{2} E\right] 2 \operatorname{Re}\left[\left(\Delta(\boldsymbol{r})-\Delta_{0}\right) \Delta_{0}{ }^{*}\right], \tilde{E} \equiv\left(\varepsilon^{2}+|\Delta(\boldsymbol{r})|^{2}\right)^{1 / 2}
\end{gathered}
$$

mit

ersetzen. Legen wir jetzt $\Delta_{0}$ durch

$$
\Delta_{0}=\frac{1}{\Omega} \int \mathrm{d}^{3} \boldsymbol{r} \Delta(\boldsymbol{r})
$$

fest, so verschwindet der lineare Term $m^{\prime}$ in Gl. (30). Benutzen wir noch die Näherungen

$|\Delta(\boldsymbol{r})|=\left|\Delta_{0}\right|+\frac{1}{\left|\Delta_{0}\right|} \operatorname{Re}\left[\left(\Delta(\boldsymbol{r})-\Delta_{0}\right) \Delta_{0}^{*}\right]+\ldots, \boldsymbol{A}(\boldsymbol{r}) \Delta(\boldsymbol{r})=\boldsymbol{A}(\boldsymbol{r}) \Delta_{0}+\ldots, \frac{\Delta^{*}(\boldsymbol{r}) \Delta\left(\boldsymbol{r}^{\prime}\right)}{|\Delta(\boldsymbol{r})|\left|\Delta\left(\boldsymbol{r}^{\prime}\right)\right|}=1+\ldots$

und ersetzen außerdem näherungsweise in den Integralkernen $\left|\Delta_{0}\right|$ durch

so erhalten wir an Stelle von Gl. (27)

$$
\Delta \equiv \frac{1}{\Omega} \int_{\Omega} \mathrm{d}^{3} \boldsymbol{r}|\Delta(\boldsymbol{r})|
$$

$$
\begin{aligned}
& M(\Delta, \boldsymbol{A})-M(0, \boldsymbol{A})=\int_{\Omega} \mathrm{d}^{3} \boldsymbol{r}\left\{\frac{1}{V_{0}}|\Delta(\boldsymbol{r})|^{2}+m\left(|\Delta(\boldsymbol{r})|^{2}\right)\right\} \\
& +2 \Delta^{2} \int_{\Omega} \mathrm{d}^{3} \boldsymbol{r} \mathrm{d}^{3} \boldsymbol{r}^{\prime}|\Delta(\boldsymbol{r})| K_{3}\left(\left|\boldsymbol{r}-\boldsymbol{r}^{\prime}\right|\right)\left|\Delta\left(\boldsymbol{r}^{\prime}\right)\right|-\frac{2 e^{2}}{m c^{2}} \int_{\Omega} \mathrm{d}^{3} \boldsymbol{r} \mathrm{d}^{3} \boldsymbol{r}^{\prime} \operatorname{rot}_{l} \boldsymbol{A}(\boldsymbol{r}) K_{2}\left(\left|\boldsymbol{r}-\boldsymbol{r}^{\prime}\right|\right) \operatorname{rot}_{l}^{\prime} \boldsymbol{A}\left(\boldsymbol{r}^{\prime}\right) \\
& +\frac{1}{2 m} \int_{\Omega} \mathrm{d}^{3} \boldsymbol{r} \mathrm{d}^{3} \boldsymbol{r}^{\prime} \frac{\Delta^{*}(\boldsymbol{r})}{|\Delta(\boldsymbol{r})|}\left[\left(\frac{\hbar}{i} \nabla_{l}-\frac{2 e}{c} \boldsymbol{A}_{l}(\boldsymbol{r})\right) \Delta(\boldsymbol{r})\right] K_{1}\left(\left|\boldsymbol{r}-\boldsymbol{r}^{\prime}\right|\right)\left[\left(-\frac{\hbar}{i} \nabla_{l}^{\prime}-\frac{2 e}{c} \boldsymbol{A}_{l}\left(\boldsymbol{r}^{\prime}\right)\right) \Delta^{*}\left(\boldsymbol{r}^{\prime}\right)\right] \frac{\Delta\left(\boldsymbol{r}^{\prime}\right)}{\left|\Delta\left(\boldsymbol{r}^{\prime}\right)\right|}
\end{aligned}
$$

$\mathrm{Zu}$ der Genauigkeit der vorangehenden Näherungen ist zu sagen, daß wir nicht so sehr an einer bezüglich des Ortes gleichmäßigen Approximation von $\Delta(\boldsymbol{r})$ und $\boldsymbol{A}(\boldsymbol{r})$ interessiert sind, als vielmehr an einer möglichst guten Approximation der Freien Energie. Lokale große Schwankungen des Ordnungsparameters, die keine Näherungen der Art von Gl.
(32 a, b, c) erlauben, sind trotzdem zugelassen, wenn ihr Energiebeitrag relativ klein ist.

Aus Gl. (34) folgen durch Variation nach Betrag und Phase des Ordnungsparameters die notwendigen Bedingungen für das Minimum von $M$. Ob tatsächlich ein Minimum vorliegt, muß am konkreten Problem durch Einsetzen der Lösung nachgeprüft wer- 
den. Der Übersichtlichkeit wegen variieren wir nur nach explizit vorkommendem $\Delta(\boldsymbol{r})$, und halten $\Delta$ [Gl. (33) ] mit einem Lagrangeschen Parameter konstant. Wir erhalten demnach gemäß

$\frac{\delta}{\delta|\Delta(\boldsymbol{r})|}\left\{M(\Delta, \boldsymbol{A})+\eta\left[\int_{\Omega} \mathrm{d}^{3} \boldsymbol{r}|\Delta(\boldsymbol{r})|-\Omega \Delta\right]\right\}=0$,

$$
\begin{gathered}
\frac{\delta}{\delta \varphi(\boldsymbol{r})} M(\Delta, \boldsymbol{A})=0, \\
\frac{\partial}{\partial \eta}\left\{M(\Delta, \boldsymbol{A})+\eta\left[\int_{\Omega} \mathrm{d}^{3} \boldsymbol{r}|\Delta(\boldsymbol{r})|-\Omega \Delta\right]\right\}=0,
\end{gathered}
$$

mit

$$
\begin{gathered}
\Delta(\boldsymbol{r}) \equiv|\Delta(\boldsymbol{r})| \exp \{i \varphi(\boldsymbol{r})\}, \\
\boldsymbol{G}(\boldsymbol{r}) \equiv \boldsymbol{A}(\boldsymbol{r})-(\hbar c / 2 e) \operatorname{grad} \varphi(\boldsymbol{r})
\end{gathered}
$$

die Variationsgleichungen

$\frac{1}{2} \frac{\partial}{\partial|\Delta(\boldsymbol{r})|}\left\{m\left(|\Delta(\boldsymbol{r})|^{2}\right)+\eta|\Delta(\boldsymbol{r})|+\frac{1}{V_{0}}|\Delta(\boldsymbol{r})|^{2}\right\}$

$=-2 \Delta_{\Omega}^{2} \int^{3} \boldsymbol{d}^{\prime} K_{3}\left(\left|\boldsymbol{r}-\boldsymbol{r}^{\prime}\right|\right)\left|\Delta\left(\boldsymbol{r}^{\prime}\right)\right|$

$+\frac{\hbar^{2}}{2 m} \operatorname{div} \int_{\Omega} \mathrm{d}^{3} \boldsymbol{r}^{\prime} K_{1}\left(\left|\boldsymbol{r}-\boldsymbol{r}^{\prime}\right|\right) \operatorname{grad}^{\prime}\left|\Delta\left(\boldsymbol{r}^{\prime}\right)\right|$

$-\frac{2 e^{2}}{m c^{2}} \boldsymbol{G}_{l}(\boldsymbol{r}) \int_{\Omega} \mathrm{d}^{3} \boldsymbol{r}^{\prime} K_{1}\left(\left|\boldsymbol{r}-\boldsymbol{r}^{\prime}\right|\right)\left|\Delta\left(\boldsymbol{r}^{\prime}\right)\right| G_{l}\left(\boldsymbol{r}^{\prime}\right)$,

$\operatorname{div}\left[|\Delta(\boldsymbol{r})| \int_{\Omega} \mathrm{d}^{3} \boldsymbol{r}^{\prime} K_{1}\left(\left|\boldsymbol{r}-\boldsymbol{r}^{\prime}\right|\right)\left|\Delta\left(\boldsymbol{r}^{\prime}\right)\right| \boldsymbol{G}\left(\boldsymbol{r}^{\prime}\right)\right]=0$,

$$
\Delta=\frac{1}{\Omega} \int_{\Omega} \mathrm{d}^{3} \boldsymbol{r}|\Delta(\boldsymbol{r})| .
$$

Für den Strom folgt aus der Definitionsgleichung (8)

$$
\begin{aligned}
& -\frac{1}{c} \boldsymbol{J}(\boldsymbol{r}) \\
& =\frac{4 e^{2}}{m c^{2}} \int_{\Omega} \mathrm{d}^{3} \boldsymbol{r}^{\prime}\left[K_{1}\left(\left|\boldsymbol{r}-\boldsymbol{r}^{\prime}\right|\right) \boldsymbol{G}\left(\boldsymbol{r}^{\prime}\right)\left|\Delta\left(\boldsymbol{r}^{\prime}\right)\right||\Delta(\boldsymbol{r})|\right. \\
& \left.\quad-\operatorname{rot}\left\{K_{2}\left(\left|\boldsymbol{r}-\boldsymbol{r}^{\prime}\right|\right) \operatorname{rot}^{\prime} \boldsymbol{A}\left(\boldsymbol{r}^{\prime}\right)\right\}\right] .
\end{aligned}
$$

Da $M$ auch bezüglich $\Delta$ stationär sein muß, ist außerdem

$$
\frac{\partial}{\partial \Delta} M(\Delta, \boldsymbol{A})=\eta \Omega
$$

zu fordern. Aus Gl. (37 b und d) folgt die Ladungserhaltung

$$
\operatorname{div} \boldsymbol{J}=0 \text {. }
$$

Mit der Maxwellschen Gleichung

$$
c \operatorname{rot} \operatorname{rot} \boldsymbol{A}=4 \pi \boldsymbol{J}
$$

bleibt also zur Beschreibung des Supraleiters im statischen Magnetfeld ein aus 6 Gleichungen (37 a, c bis $37 \mathrm{~g}$ ) bestehendes nichtlineares System von Integrodifferentialgleichungen bezüglich der Größen $|\Delta(\boldsymbol{r})|, \varphi(\boldsymbol{r}), \boldsymbol{A}(\boldsymbol{r}), \boldsymbol{J}(\boldsymbol{r}), \eta$ und $\Delta$ zu lösen. Dabei sind vier Größen Funktionen des Ortes.

Für die Lösung bietet sich als Spezialfall die Linearisierung des Gleichungssystems an. Man approximiert nach einer fest vorgegebenen Eichung die ortsabhängigen Parameter teilweise durch ihre Mittelwerte, so daß explizit ortsabhängige Unbekannte nur noch linear in den Gleichungen vorkommen, obwohl die Theorie natürlich wegen der anderen Parameter weiterhin nichtlinear bleibt.

\section{Ginzburg-Landau-Theorie}

Für den Fall schwacher Ortsabhängigkeit werden die Integralkerne nur in der Grenze $\boldsymbol{q} \rightarrow 0$ benötigt. Die Entwicklung um $\boldsymbol{q}=0$ ergibt

$$
\begin{gathered}
K_{1}(\boldsymbol{q})=\frac{n}{4}\left(1+\frac{\left(\pi q \xi_{0} \varepsilon_{0}\right)^{2}}{10} \frac{\partial}{\partial \Delta^{2}}\right) \frac{\Lambda_{0}}{\Delta^{2} \Lambda} \\
=\frac{m c^{2}}{16 \pi e^{2}}\left(1+\frac{\left(\pi q \xi_{0} \varepsilon_{0}\right)^{2}}{10} \frac{\partial}{\partial \Delta^{2}}\right) \frac{1}{\Delta^{2} \lambda^{2}}, \\
K_{2}(\boldsymbol{q})=\frac{n\left(\xi_{0} \Delta \pi \varepsilon_{0}\right)^{2}}{60} \frac{\partial}{\partial \Delta^{2}} \frac{\Lambda_{0}}{\Delta^{2} \Lambda}, \\
K_{3}(\boldsymbol{q})=\frac{n \hbar^{2} q^{2}}{24 m} \frac{\partial}{\partial \Delta^{2}} \frac{\Lambda_{0}}{\Delta^{2} \Lambda} .
\end{gathered}
$$

Die in Gl. (38 a) erscheinende Eindringtiefe $\lambda$ ist, wenn $\Delta$ durch die BCS-Energielücke $\varepsilon_{\mathrm{T}}$ ersetzt wird, mit der Londonschen Eindringtiefe $\lambda_{\mathrm{T}}$ für schwache Felder identisch. Verlangt man, daß $\left(|\Delta(\boldsymbol{r})|-\varepsilon_{\mathrm{T}}\right)$ klein ist, so kann man in Gl. (34) $|\Delta(\boldsymbol{r})|$ um $\varepsilon_{\mathrm{T}}$ entwickeln. Wegen Gl. $(38 \mathrm{~b}, \mathrm{c})$ ergeben $K_{2}$ und $K_{3}$ erst in höherer Ordnung einen Beitrag. Es bleibt nur der erste und letzte Term auf der rechten Seite von Gl. (34) übrig, und es folgt mit Gl. (38 a) das Funktional der Ginzburg-Landau-Theorie

$$
\begin{aligned}
& M_{\mathrm{G} . \mathrm{L} .}(\Delta, \boldsymbol{A})-M_{\mathrm{G} . \mathrm{L} .}(0, \boldsymbol{A}) \\
& =\int \mathrm{d}^{3} \boldsymbol{r}\left\{\left(1+\left[|\Delta(\boldsymbol{r})|^{2}-\varepsilon_{\mathrm{T}}^{2}\right] \frac{\partial}{\partial \varepsilon_{\mathrm{T}}^{2}}\right)\left(m\left(\varepsilon_{\mathrm{T}}^{2}\right)+\frac{\varepsilon_{\mathrm{T}}^{2}}{V_{0}}\right)\right. \\
& \quad+\frac{1}{2}\left[|\Delta(\boldsymbol{r})|^{2}-\varepsilon_{\mathrm{T}}^{2}\right]^{2} \frac{\partial^{2}}{\partial\left(\varepsilon_{\mathrm{T}}^{2}\right)^{2}} m\left(\varepsilon_{\mathrm{T}}^{2}\right) \\
& \left.+\frac{c^{2}}{32 \pi e^{2} \varepsilon_{\mathrm{T}}^{2} \lambda_{\mathrm{T}}^{2}}\left|\left(\frac{\hbar}{i} \nabla-\frac{2 e}{c} \boldsymbol{A}\right) \Delta(\boldsymbol{r})\right|^{2}\right\} .
\end{aligned}
$$




\section{Randbedingungen}

Das Gleichungssystem (37) ist unter periodischen Randbedingungen für sehr großes Normierungsvolumen $\Omega$ abgeleitet worden. Für physikalische Systeme sind jene allerdings nicht zu gebrauchen.

Ist das Gleichungssystem, wie schon beschrieben, linearisiert, so kann man Spiegelung als Randbedingung einführen, indem man die Gültigkeit des Gleichungssystems nur im Innern des massiven Supraleiters fordert und an der Oberfläche das Verschwinden der Normalkomponente von

$$
[(\hbar / i) \nabla-(2 e / c) \boldsymbol{A}] \Delta(\boldsymbol{r})
$$

verlangt ${ }^{6}$. Darauf soll hier nicht näher eingegangen werden.

Statt dessen verschaffen wir uns Randbedingungen für "diffuse Streuung“, indem wir in dem Funktional der Gl. (34) außerhalb des vom Supraleiter ausgefüllten Raumes

$$
|\Delta(\boldsymbol{r})| \equiv 0
$$

oder

$$
\operatorname{grad}|\Delta(\boldsymbol{r})| \equiv 0
$$

setzen. Der letzte Fall entspricht einer diffusen Streuung der Cooper-Paare an der Oberfläche, der erste einer Paarbrechung. Ebenso wird außen

$$
[\boldsymbol{A}(\boldsymbol{r})-(\hbar c / 2 e) \operatorname{grad} \varphi(\boldsymbol{r})] \equiv 0
$$

gesetzt, wie es für $\varphi(\boldsymbol{r}) \equiv$ const in der linearen Theorie bei diffus streuender Oberfläche üblich ist ${ }^{7}$.

Die Bedeutung von Gl. (40) folgt anschaulich daraus, daß bei einem unendlich ausgedehnten $\mathrm{Su}$ praleiter in dem Bereiche mit $|\Delta(\boldsymbol{r})| \equiv 0$ fest vorgegeben sind, die Grenzflächen zwischen diesen Bereichen und dem übrigen supraleitenden Material paarbrechende Eigenschaften haben müssen. Da aber der Volumenanteil der normalleitenden Bereiche in dem Funktional Gl. (34) keinen Beitrag liefert, oder anders ausgedrückt, den gleichen Beitrag liefert wie ein entsprechendes materiefreies Volumen, verbleibt nur die Wirkung der Grenzfläche als paarbrechende Oberfläche eines endlichen Supraleiters. Die Bindung der Elektronen, die von der Oberfläche gestreut werden, wird aufgebrochen, so daß sie die gleiche Information besitzen, wie dann, wenn sie wirklich aus einem Gebiet mit $|\Delta(\boldsymbol{r})| \equiv 0$ kommen.

Eine Aussage über die Randbedingung (41) erhält man auf ähnlichem Wege. Die Oberfläche redu-

\footnotetext{
${ }^{6}$ M. R. Schafroth, Theoretical Aspects of Superconductivity, in Solid State Physics, Academic Press Inc., New York 1960, Bd. 10, p. 293.
}

ziert die Schwerpunktsbewegung der ankommenden Cooper-Paare auf Null, so als ob sie in einen Bereich gelangen, in dem der Ordnungsparameter konstant ist. Sie verhält sich für die auftreffenden Paare wie eine unelastische Wand. Die Elektronen verlieren also an der Oberfläche die Information über die Ortsabhängigkeit des Ordnungsparameters in einer infinitesimalen Umgebung der Oberfläche.

Formal geht man folgendermaßen vor. Je nachdem ob Gl. (40) oder Gl. (41) gefordert wird, ist das Funktional (34) durch partielle Integrationen in Abhängigkeit von $|\Delta(\boldsymbol{r})|$ bzw. $\operatorname{grad}|\Delta(\boldsymbol{r})|$ zu schreiben. Die Oberflächenintegrale verschwinden wegen der periodischen Randbedingungen. Dann erst werden durch Anwendung einer der beiden Gleichungen die Integrationen auf den tatsächlichen Bereich des Supraleiters beschränkt. Das so entstehende Funktional wird minimalisiert, wobei die Randwerte von $|\Delta(\boldsymbol{r})|$ bei der Variation festzuhalten sind. Das führt auf ein Gleichungssystem, das den jeweiligen Randbedingungen angepaßt ist. Man kann, indem man in Gl. (34) die von $|\Delta(\boldsymbol{r})|$ abhängenden Terme mit Hilfe eines neuen Parameters linear aufteilt, beliebige Kombinationen zwischen (40) und (41) schaffen. In dem Beispiel des nächsten Teils bedeutet dieser Parameter die relative Häufigkeit der Paarbrechung an der Oberfläche.

Oberflächen, die Supraleiter und Dielektrikum trennen, sollten sich mit der Randbedingung der Gl. (41) beschreiben lassen. Metallische normalleitende Aufdampfschichten ergeben je nach Dicke und Art einen mehr oder weniger starken Paarbrechungseffekt, was durch eine Oberfläche, die zum Teil der durch Gl. (40) definierten Voraussetzung genügt, simuliert werden kann. Man verzichtet bei diesem Vorgehen auf Feinheiten in der Oberfläche selbst, man erhält aber ihren Gesamteinfluß auf den Supraleiter.

Im folgenden werden am Beispiel der supraleitenden Platte die Randbedingungen untersucht.

\section{E. Supraleitende ebene Schicht}

Wir legen die Schicht so, daß die $x$-Koordinate auf der Oberfläche senkrecht steht und der Koordinatenanfangspunkt die Mitte der Schicht kennzeichnet

7 J. Bardeen, Theory of Superconductivity, in Handbuch der Physik, Springer-Verlag, Berlin 1956, Bd. XV, p. 274. 
( $-\frac{1}{2} d \leqq x \leqq+\frac{1}{2} d, d=$ Dicke der Schicht). In $y$ und $z$-Richtung behalten wir die periodischen Randbedingungen bei. Da dieses Beispiel nur einer qualitativen Untersuchung der Randbedingungen dienen soll, verzichten wir auf den Einfluß eines Magnetfeldes und setzen $\boldsymbol{A}(\boldsymbol{r}) \equiv 0, \varphi(\boldsymbol{r}) \equiv 0$. Aus dem gleichen Grunde beschränken wir uns auf die Umgebung eines Phasenübergangs zweiter Ordnung.

Die Freie Energie des auch in $x$-Richtung unendlich ausgedehnten Supraleiters wird in der Form [siehe Gln. (28), (34)]

$$
\begin{aligned}
& M(\Delta, 0)-M(0,0)=L_{-L / 2}^{+L / 2} \mathrm{~d} x\left\{\frac{1}{V_{0}}|\Delta(x)|^{2}+m\left(|\Delta(x)|^{2}\right)\right\} \\
& \qquad \int_{-L / 2}^{+L / 2} \mathrm{~d} x \mathrm{~d} x^{\prime}\left\{2 \Delta^{2}|\Delta(x)| K_{3}\left(\left|x-x^{\prime}\right|\right)\left|\Delta\left(x^{\prime}\right)\right|+\frac{\hbar^{2}}{2 m} \frac{\mathrm{d}|\Delta(x)|}{\mathrm{d} x} K_{1}\left(\left|x-x^{\prime}\right|\right) \frac{\mathrm{d}\left|\Delta\left(x^{\prime}\right)\right|}{\mathrm{d} x^{\prime}}\right\} \\
& \text { mit } \\
& \qquad K_{n}\left(\left|x-x^{\prime}\right|\right) \equiv \frac{1}{L} \sum_{\boldsymbol{q}_{x}} K_{n}\left(\boldsymbol{q}_{x}, \boldsymbol{q}_{y}=0, \boldsymbol{q}_{z}=0\right) \exp \left\{-i \boldsymbol{q}_{x}\left(x-x^{\prime}\right)\right\}
\end{aligned}
$$

mit

geschrieben. Die Integrationen erstrecken sich über die Kantenlänge $L$ des Normierungsvolumens $\Omega=L^{3}$. Da am Phasenübergang zweiter Ordnung $|\Delta(x)|$ klein ist, kann wegen Gl. (29 c) und (33) der Term mit $K_{3}\left(\left|x-x^{\prime}\right|\right)$ vernachlässigt werden. In das erste Integral auf der rechten Seite von Gl. (42) setzen wir die Ginzburg-Landau-Näherung in niedrigster Ordnung ein.

$$
\frac{1}{V_{0}}|\Delta(x)|^{2}+m\left(|\Delta(x)|^{2}\right) \approx-\frac{H_{\mathrm{cb}}^{2}}{4 \pi \varepsilon_{\mathrm{T}}^{2}}|\Delta(x)|^{2} \quad\left(H_{\mathrm{cb}} \text { Thermodyn. krit. Feld }\right) .
$$

Es folgt

$$
\begin{array}{r}
\frac{1}{L^{2}}[M(\Delta, 0)-M(0,0)]=-\frac{H_{\mathrm{cb}}^{2}}{4 \pi \varepsilon_{T}^{2}} \int_{-L / 2}^{+L / 2} \mathrm{~d} x|\Delta(x)|^{2}-p \frac{\hbar^{2}}{2 m} \int_{-L / 2}^{+L / 2} \mathrm{~d} x \mathrm{~d} x^{\prime}|\Delta(x)|\left[\frac{\partial^{2}}{\partial x^{2}} K_{1}\left(\left|x-x^{\prime}\right|\right)\right]\left|\Delta\left(x^{\prime}\right)\right| \\
+(1-p) \frac{\hbar^{2}}{2 m} \int_{-L / 2}^{+L / 2} \mathrm{~d} x \mathrm{~d} x^{\prime} \frac{\mathrm{d}|\Delta(x)|}{\mathrm{d} x} K_{1}\left(\left|x-x^{\prime}\right|\right) \frac{\mathrm{d}\left|\Delta\left(x^{\prime}\right)\right|}{\mathrm{d} x^{\prime}} .
\end{array}
$$

Gl. (45) gilt für beliebiges $p$. Der erste Ausdruck auf der rechten Seite von Gl. (45) bedeutet phänomenologisch die Selbstenergie des Volumenelements $\mathrm{d} x$ am Orte $x$ und ist demgemäß beim endlichen Supraleiter auf $-\frac{1}{2} d \leqq x \leqq+\frac{1}{2} d$ zu beschränken. Der zweite und dritte Ausdruck beschreibt die Wechselwirkung der Volumenelemente untereinander. Die Größe $p, 0 \leqq p \leqq 1$, soll die Wahrscheinlichkeit sein, daß ein Elektron so an der Wand gestreut wird, daß es aus einem Gebiet mit $|\Delta(x)| \equiv 0$ zu kommen scheint. Mit der relativen Häufigkeit $p$ werden also Cooper-Paare an der Wand aufgebrochen; $p$ ist damit der Bruchteil der Wechselwirkungsenergie, in dem im Außengebiet der Ordnungsparameter selbst verschwindet. Mit einem Anteil $(1-p)$ werden die Cooper-Paare diffus gestreut, d. h. in einem Bruchteil $(1-p)$ der Wechselwirkung ist außen $\frac{\mathrm{d}}{\mathrm{d} x}|\Delta(x)| \equiv 0$ zu setzen.

Daher sind in Gl. (45) sämtliche Integrationen auf den Bereich $-\frac{1}{2} d \leqq x \leqq+\frac{1}{2} d$ zu beschränken. Die Minimalisierung bezüglich $|\Delta(x)|$ liefert die Variationsgleichung

$$
\begin{aligned}
\frac{H_{\mathrm{cb}}^{2}}{2 \pi \varepsilon_{\mathrm{T}}^{2}}|\Delta(x)|+\frac{\hbar^{2}}{m} \int_{-d / 2}^{+d / 2} \mathrm{~d} x^{\prime} & {\left[\frac{\partial^{2}}{\partial x^{2}} K_{1}\left(\left|x-x^{\prime}\right|\right)\right]\left|\Delta\left(x^{\prime}\right)\right| } \\
& \left.=(p-1) \frac{\hbar^{2}}{m}\left|\frac{\partial}{\partial x} K_{1}(|x-d / 2|)\right| \Delta(d / 2)\left|-\frac{\partial}{\partial x} K_{1}(|x+d / 2|)\right| \Delta(-d / 2) \mid\right] .
\end{aligned}
$$


Für die Integralkerne kann man einfache Näherungen finden. Aus Gl. (29 a) gewinnt man die asymptotische Darstellung

$$
K_{1}(\boldsymbol{q})=\frac{3 n}{\left(\pi q \xi_{0} \varepsilon_{0}\right)^{2}} \log \frac{\pi q \xi_{0}}{e t}+\ldots, \quad\left(q \xi_{0}\right) \gg 1 .
$$

Gl. (38 a) liefert mit $\Delta / k T \ll 1$

$$
K_{1}(\boldsymbol{q})=\frac{n \lambda(3)}{2\left(\gamma t \varepsilon_{0}\right)^{2}}\left\{1-\frac{3 \lambda(5)}{20 \lambda(3)}\left(\frac{\pi q \xi_{0}}{\gamma t}\right)^{2}+\ldots\right\}, \quad\left(q \xi_{0}\right) \ll 1 .
$$

Dabei wurde

$$
\begin{aligned}
& T / T_{\mathrm{c}} \equiv t, \quad T_{\mathrm{c}} \text { Sprungtemperatur des massiven Supraleiters, } \\
& \lambda(n) \equiv \sum_{k=0}^{\infty}(2 k+1)^{-n}, \quad \gamma \equiv e^{C} \equiv 1,781 \ldots C \text { Eulersche Konst. }
\end{aligned}
$$

gesetzt. Die Näherung

$$
K_{1}(\boldsymbol{q})=\frac{n \lambda(3)}{2\left(\gamma t \varepsilon_{0}\right)^{2}} \frac{1}{1+\frac{1}{6} \lambda(3)\left(\pi q \xi_{0} / \gamma t\right)^{2}\left[\log \left\{3+\left(\pi q \overline{\xi_{0}} / e t\right)\right\}\right]^{-1}}
$$

stimmt in den entsprechenden Grenzfällen mit Gl. (47) bzw. Gl. (48) in den ausgeschriebenen Ordnungen überein. Den Logarithmus in Gl. (49) betrachten wir als schwach veränderliche Funktion und setzen im Argument $\boldsymbol{q}_{x}=$ const $/ d$, weil für kleinere $d$ immer größere Werte von $\boldsymbol{q}_{x}$ wichtig werden. Für die unter diesen Annahmen berechnete Lösung der Integralgleichung (46) kann die Freie Energie mit dem exakten Integralkern in der Grenze $d \rightarrow 0$ bestimmt werden. Der Vergleich mit der Freien Energie, die den genäherten Integralkern enthält, liefert die Konstante und damit die Rechtfertigung für den Ansatz. Wir nehmen das vorweg und ersetzen $\boldsymbol{q}_{x}$ durch $e / \gamma d$.

$$
\log \left(3+\frac{\pi\left|\boldsymbol{q}_{x}\right| \xi_{0}}{e t}\right) \approx \log \left(3+\frac{\pi \xi_{0}}{\gamma d t}\right) \equiv f(d t) .
$$

Mit Gl. (49) und Gl. (50) erhalten wir einen einfach zu handhabenden Integralkern

$$
\begin{gathered}
K_{1}\left(\left|x-x^{\prime}\right|\right)=a b e^{-b\left|x-x^{\prime}\right|}, \\
a \equiv \frac{n \lambda(3)}{4\left(\gamma t \varepsilon_{0}\right)^{2}}, \quad b \equiv \frac{\gamma t}{\pi \xi_{0}}\left[\frac{6 f(d t)}{\lambda(3)}\right]^{1 / 2} \\
\frac{\partial^{2}}{\partial x^{2}} K_{1}\left(\left|x-x^{\prime}\right|\right)=-2 a b^{2} \delta\left(x-x^{\prime}\right)+a b^{3} e^{-b\left|x-x^{\prime}\right|}
\end{gathered}
$$

$$
\left(\delta\left(x-x^{\prime}\right) \text { Dirac } \delta\right. \text {-Funktion). }
$$

Gl. (46) hat damit die Lösung

$$
|\Delta(x)|=\Delta \cos (\mu x) \frac{\mu d}{2 \sin \left(\frac{1}{2} \mu d\right)}
$$

mit

$$
\mu \operatorname{tg}\left(\frac{1}{2} \mu d\right)=\left(\mu^{2} / b\right)[p-1]+p b
$$

und

$$
\mu^{2}=b^{2}\left[\left(\frac{H_{\mathrm{cb}}(0) \varepsilon_{\mathrm{T}}}{H_{\mathrm{cb}}(T) \varepsilon_{0}}\right)^{2} f(d t)-1\right]^{-1} .
$$

Aus Gl. (54) und Gl. (55) folgt $\mu$ und die Sprungtemperatur $T_{\mathrm{c}}{ }^{\prime}$ der Schicht. Die numerische Lösung der beiden Gleichungen führt auf die Kurven in Abb. 1. Die Funktionen $H_{\mathrm{cb}}(T)$ und $\varepsilon_{\mathrm{T}}$ wurden der BCS-Theorie entnommen ${ }^{8}$. Die Sprungtemperatur der Schicht nimmt mit der Dicke ab. Der linke Endpunkt kennzeichnet die Dicke, unterhalb der im Rahmen dieser Ableitung kein Phasenübergang zweiter Ordnung möglich ist. Die Wirkung der Oberfläche ist dann vermutlich im Verhältnis zur geringen Dicke der Schicht zu groß, um sie noch als kleine Störung behandeln zu dürfen.

Ähnliche Kurven, wie die in Abb. 1, erhält man auch aus dem Proximity-Effekt ${ }^{9}$, der daher zur Eichung des Parameters $p$ in Abhängigkeit von der Art der metallischen Auflage dienen kann.

Auf diese Weise hat man mit $p$ die Randbedingungen auch außerhalb der nächsten Umgebung des Phasenüberganges festgelegt. Das ist der eigentliche Zweck dieses Beispiels, besonders da Randbedingungen, solange man nur auf den Phasenübergang selbst

8 B. Mühlschlegel, Z. Phys. 155, 313 [1959].

9 P. Hilsch u. R. Hilsch, Z. Phys. 180, 10 [1964]. Der Vergleich mit Fig. 3 jener Arbeit zeigt, daß die kritische Dicke $D_{\mathrm{s} 0}$ in Beziehung zu $p$ gesetzt werden kann:

$$
D_{\mathrm{s} 0}(p=0,1) \approx 0,02 \quad \xi_{0}, \quad D_{\mathrm{s} 0}(p=0,5) \approx 0,8 \xi_{0} .
$$


Wert legt, über die Methode der Korrelationsfunktion gewonnen werden können ${ }^{10}$.

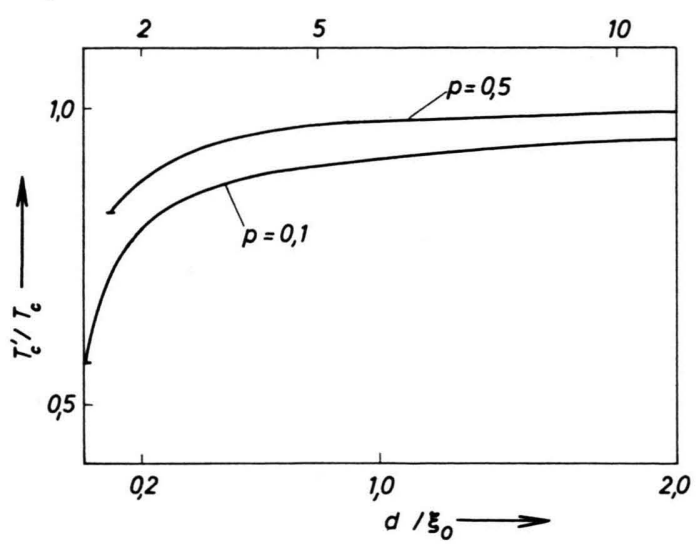

Abb. 1. Sprungtemperatur als Funktion der Dicke der Schicht (obere Abszisse $p=0,5$ ).

Es sei noch darauf hingewiesen, daß sich die Integralgleichung (45) als Differentialgleichung, ähnlich der Ginzburg-Landau-Gleichung, schreiben läßt

$$
\frac{\mathrm{d}^{2}}{\mathrm{~d} x^{2}}|\Delta(x)|+\mu^{2}|\Delta(x)|=0
$$

mit den Randbedingungen

$$
\begin{aligned}
\pm \frac{\mathrm{d}}{\mathrm{d} x}|\Delta(x)| & = \\
& -\left.\left(\frac{\mu^{2}}{b}[p-1]+b p\right)|\Delta(x)|\right|_{x= \pm d / 2}
\end{aligned}
$$

Ist die Sprungtemperatur $T_{\mathrm{c}}{ }^{\prime}$ ungefähr gleich $T_{\mathrm{c}}$ oder $d$ sehr groß, so folgt aus $\mathrm{Gl}$. (55)

$$
\begin{gathered}
\mu^{2} \rightarrow \varkappa^{2} / \lambda_{\mathrm{T}}{ }^{2} \rightarrow 0 \text { für } T=T_{\mathrm{c}}{ }^{\prime} \rightarrow T_{\mathrm{c}} ; \\
\varkappa^{2}=\left(8 e^{2} / \hbar^{2} c^{2}\right) \lambda_{\mathrm{T}}^{4} H_{\mathrm{Ch}}^{2}(T) .
\end{gathered}
$$

Gl. (56) ist dann in diesem einfachen Fall mit der entsprechenden Ginzburg-Landau-Gleichung identisch. Letzteres gilt auch für die Randbedingung (57) im Fall $p \rightarrow 0$. De Gennes diskutiert im Zusammenhang mit der Korrelationsfunktion eine formal ähnliche Randbedingung ${ }^{10}$.

Herrn Prof. Dr. H. Koppe danke ich herzlich für die Anregung zu dieser Arbeit, sowie für viele hilfreiche Diskussionen. - Diese Arbeit wurde zum Teil durch eine Sachbeihilfe der Deutschen Forschungsgemeinschaft unterstützt. - Für die numerische Rechnung stand mir Rechenzeit vom Rechenzentrum der Universität Kiel zur Verfügung.

\section{Anhang: Berechnung der Freien Energie}

Die Greenschen Funktionen, die auf der rechten Seite von Gl. (25) erscheinen, lassen sich folgendermaßen darstellen ${ }^{11}$

$$
\begin{gathered}
G_{12}=\frac{1}{\Omega} \sum_{\boldsymbol{k}} \exp \left\{i \boldsymbol{k}\left(\boldsymbol{r}_{1}-\boldsymbol{r}_{2}\right)\right\} \frac{\operatorname{sign}\left(\beta_{1}-\beta_{2}\right) \cosh (x E)+(\varepsilon / E) \sinh (x E)}{2 \cosh \left(\frac{1}{2} \beta E\right)}, \\
F_{12}=\frac{1}{\Omega} \sum_{\boldsymbol{k}} \exp \left\{-i \boldsymbol{k}\left(\boldsymbol{r}_{1}-\boldsymbol{r}_{2}\right)\right\} \frac{\Delta_{0} \sinh (x E)}{2 E \cosh \left(\frac{1}{2} \beta E\right)}, \quad F_{12}^{+}=F_{12}^{*}=\left(\Delta_{0}^{*} / \Delta_{0}\right) F_{12}
\end{gathered}
$$

mit

$$
x=\frac{1}{2} \beta-\left|\beta_{1}-\beta_{2}\right|, E=\left(\varepsilon^{2}+\left|\Delta_{0}\right|^{2}\right)^{1 / 2} .
$$

Die $\beta$-Integrationen in Gl. (25) können elementar ausgeführt werden.

$$
\begin{aligned}
\int_{0}^{\beta} \mathrm{d} \beta_{1} \ldots \mathrm{d} \beta_{2}^{\prime} & \delta\left(\beta_{1}-\beta_{1}^{\prime}\right) \delta\left(\beta_{2}-\beta_{2}^{\prime}\right)\left[F_{1^{\prime} 2^{\prime}}^{+} F_{12}-G_{21^{\prime}} G_{12^{\prime}}\right]= \\
& +\frac{\beta}{2 \Omega^{2}} \sum_{k k^{\prime}} \frac{\exp \left\{-i \boldsymbol{k} \boldsymbol{r}_{1}^{\prime}+i \boldsymbol{k}^{\prime} \boldsymbol{r}_{1}\right\}}{\varepsilon^{2}-\varepsilon^{\prime 2}} \exp \left\{i \boldsymbol{k} \boldsymbol{r}_{2}^{\prime}-i \boldsymbol{k}^{\prime} \boldsymbol{r}_{2}\right\}\left|\Delta_{0}\right|^{2}\left\{\frac{1}{E^{\prime}} \tanh \left(\frac{1}{2} \beta E^{\prime}\right)\right. \\
& \left.\left.-\frac{1}{E} \tanh \left(\frac{1}{2} \beta E\right)\right\}-\exp \left\{i \boldsymbol{k} \boldsymbol{r}_{2}-i \boldsymbol{k}^{\prime} \boldsymbol{r}_{2}^{\prime}\right\}\left\{\left(E^{\prime}+\frac{\varepsilon \varepsilon^{\prime}}{E^{\prime}}\right) \tanh \left(\frac{1}{2} \beta E^{\prime}\right)-\left(E+\frac{\varepsilon \varepsilon^{\prime}}{E}\right) \tanh \left(\frac{1}{2} \beta E\right)\right\}\right]
\end{aligned}
$$

$$
\begin{aligned}
& \int_{0}^{\beta} \mathrm{d} \beta_{1} \ldots \mathrm{d} \beta_{2} \delta\left(\beta_{1}-\beta_{1}^{\prime}\right)\left[\Delta_{2}\left(F_{1^{\prime 2}}^{+}+F_{21^{\prime}}^{+}\right) G_{12}+\Delta_{2}{ }^{*}\left(F_{12}+F_{21}\right) G_{21^{\prime}}\right]= \\
& +\frac{\beta}{\Omega^{2}} \sum_{\boldsymbol{k} \boldsymbol{k}^{\prime}} \varepsilon \frac{\exp \left\{-i \boldsymbol{r}_{2}\left(\boldsymbol{k}-\boldsymbol{k}^{\prime}\right)\right\}}{\varepsilon^{2}-\varepsilon^{\prime 2}}\left[\frac{1}{E^{\prime}} \tanh \left(\frac{1}{2} \beta E^{\prime}\right)-\frac{1}{E} \tanh \left(\frac{1}{2} \beta E\right)\right]\left[\exp \left\{i \boldsymbol{k} \boldsymbol{r}_{1}-i \boldsymbol{k}^{\prime} \boldsymbol{r}_{1}{ }^{\prime}\right\} \Delta_{2} \Delta_{0}{ }^{*}\right. \\
& \left.+\exp \left\{i \boldsymbol{k} \boldsymbol{r}_{1}{ }^{\prime}-i \boldsymbol{k}^{\prime} \boldsymbol{r}_{1}\right\} \Delta_{2}{ }^{*} \Delta_{0}\right], \quad \text { (A 5) }
\end{aligned}
$$

11 Für die auftretenden Erwartungswerte s. z. B.: B. Mühlschlegel, Statistische Methode in der Theorie der Supraleitung, Verlag der Bayer. Akademie der Wissenschaften, München 1960, S. 130. 


$$
\begin{aligned}
& -\int_{0}^{\beta} \mathrm{d} \beta_{1} \mathrm{~d} \beta_{2}\left[\Delta_{1} \Delta_{2}\left(F_{12}^{+}\right)^{2}-2 \Delta_{1}{ }^{*} \Delta_{2}\left(G_{12}\right)^{2}+\Delta_{1}{ }^{*} \Delta_{2}{ }^{*}\left(F_{12}\right)^{2}\right]= \\
& =+\frac{\beta}{2 \Omega^{2}} \sum_{k k^{\prime}} \frac{\exp \left\{-i\left(\boldsymbol{k}-\boldsymbol{k}^{\prime}\right)\left(\boldsymbol{r}_{1}-\boldsymbol{r}_{2}\right)\right\}}{\varepsilon^{2}-\varepsilon^{\prime 2}}\left[\left\{-\Delta_{1} \Delta_{2} \Delta_{0}{ }^{* 2}-\Delta_{1}{ }^{*} \Delta_{2}{ }^{*} \Delta_{0}{ }^{2}\right\}\left(\frac{1}{E^{\prime}} \tanh \left(\frac{1}{2} \beta E^{\prime}\right)-\frac{1}{E} \tanh \left(\frac{1}{2} \beta E\right)\right)\right. \\
& \left.+2 \Delta_{1}{ }^{*} \Delta_{2}\left\{\left(\frac{\varepsilon \varepsilon^{\prime}}{E^{\prime}}-E^{\prime}\right) \tanh \left(\frac{1}{2} \beta E^{\prime}\right)-\left(\frac{\varepsilon \varepsilon^{\prime}}{E}-E\right) \tanh \left(\frac{1}{2} \beta E\right)\right\}\right] \quad\left(\Delta_{1}=\Delta\left(\boldsymbol{r}_{1}\right)-\Delta_{0}, \Delta_{2}=\Delta\left(\boldsymbol{r}_{2}\right)-\Delta_{0}\right) .
\end{aligned}
$$

Mit

$$
-\frac{k T}{\Omega} \log \operatorname{Spur} e^{-\beta H_{0}{ }^{0}}=-\frac{1}{\Omega}\left[\sum_{|\varepsilon|<\hbar \omega}\left(E-\varepsilon+2 k T \log \left(1+e^{-\beta E}\right)\right)+2 k T \sum_{|\varepsilon|>\hbar \omega} \log \left(1+e^{-\beta \varepsilon}\right)\right]+\frac{1}{V_{0}}|\Delta(\boldsymbol{r})|^{2}
$$

erhalten wir für $M(\Delta, \boldsymbol{A})$

$$
\begin{aligned}
& M(\Delta, \boldsymbol{A}) \equiv M_{0}+M_{1}+M_{2}+M_{3}+M_{4}, \\
& M_{0}=\frac{\Omega}{V_{0}} \sum_{\boldsymbol{q}}|\Delta(\boldsymbol{q})|^{2}-\sum_{|\varepsilon|<\hbar \omega}\left(E-\varepsilon+2 k T \log \left(1+e^{-\beta E}\right)\right)-2 k T \sum_{|\varepsilon|>\hbar \omega} \log \left(1+e^{-\beta \varepsilon}\right), \\
& M_{1}=\frac{e^{2}\langle N\rangle_{0,0}}{2 m c^{2}} \sum_{\boldsymbol{q}}|\boldsymbol{A}(\boldsymbol{q})|^{2}-\frac{1}{2}\left(\frac{e \hbar}{m c}\right)^{2} \sum_{\boldsymbol{k} \boldsymbol{q}} \frac{\left[\left(\boldsymbol{k}-\frac{1}{2} \boldsymbol{q}\right) \boldsymbol{A}(\boldsymbol{q})\right]\left[\left(\boldsymbol{k}-\frac{1}{2} \boldsymbol{q}\right) \boldsymbol{A}^{*}(\boldsymbol{q})\right]}{\varepsilon^{2}-\varepsilon^{\prime 2}}\left[\left(E+\frac{\varepsilon \varepsilon^{\prime}+\left|\Delta_{0}\right|^{2}}{E}\right)\right. \\
& \left.\times \tanh \left(\frac{1}{2} \beta E\right)-\left(E^{\prime}+\frac{\varepsilon \varepsilon^{\prime}+\left|\Delta_{0}\right|^{2}}{E^{\prime}}\right) \tanh \left(\frac{1}{2} \beta E^{\prime}\right)\right], \\
& M_{2}=-\frac{e \hbar}{m c} \sum_{k \boldsymbol{q}} \frac{\varepsilon}{\varepsilon^{2}-\varepsilon^{\prime 2}}\left[\Delta_{0}^{*} \Delta(\boldsymbol{q})\left(\boldsymbol{A}^{*}(\boldsymbol{q})\left(\boldsymbol{k}-\frac{1}{2} \boldsymbol{q}\right)\right)+\text { c.c. }\right]\left[\frac{1}{E^{\prime}} \tanh \left(\frac{1}{2} \beta E^{\prime}\right)-\frac{1}{E} \tanh \left(\frac{1}{2} \beta E\right)\right] \\
& M_{3}=-\frac{1}{2} \sum_{\boldsymbol{k q}} \frac{\left|\Delta(\boldsymbol{q})-\Delta_{0} \delta_{\boldsymbol{q} .0}\right|^{2}}{\varepsilon^{2}-\varepsilon^{\prime 2}}\left[\left(E-\frac{\varepsilon \varepsilon^{\prime}}{E}\right) \tanh \left(\frac{1}{2} \beta E\right)-\left(E^{\prime}-\frac{\varepsilon \varepsilon^{\prime}}{E^{\prime}}\right) \tanh \left(\frac{1}{2} \beta E^{\prime}\right)\right], \\
& M_{4}=\frac{1}{4} \sum_{\boldsymbol{k} \boldsymbol{q}} \frac{1}{\varepsilon^{2}-\varepsilon^{\prime 2}}\left[\Delta_{0}^{* 2}\left(\Delta(\boldsymbol{q})-\Delta_{0} \delta_{\boldsymbol{q}, 0}\right)\left(\Delta(-\boldsymbol{q})-\Delta_{0} \delta_{\boldsymbol{q}, 0}\right)+\text { c.c }\right]\left[\frac{1}{E^{\prime}} \tanh \left(\frac{1}{2} \beta E^{\prime}\right)-\frac{1}{E} \tanh \left(\frac{1}{2} \beta E\right)\right]
\end{aligned}
$$

Hierbei wurden die transformierten Größen

$$
\Delta(\boldsymbol{q})=\frac{1}{\Omega} \int_{\Omega} \mathrm{d}^{3} \boldsymbol{r} \exp \{-i \boldsymbol{q} \boldsymbol{r}\} \Delta(\boldsymbol{r}), \quad \boldsymbol{A}(\boldsymbol{q})=\frac{1}{\Omega} \int_{\Omega} \mathrm{d}^{3} \boldsymbol{r} \exp \{-i \boldsymbol{q} \boldsymbol{r}\} \boldsymbol{A}(\boldsymbol{r})
$$

benutzt. $\Omega$ ist das Periodizitätsvolumen. $\varepsilon$ und $\varepsilon^{\prime}$ stehen für $\varepsilon_{\boldsymbol{k}}$ und $\varepsilon_{\boldsymbol{k}^{\prime}}=\varepsilon_{\boldsymbol{k}-\boldsymbol{q}}$.

Ziehen wir von $M(\Delta, \boldsymbol{A})$ den Beitrag des Normalleiters $M(0, \boldsymbol{A})$ ab, so ändert sich an der Variationsgleichung für $\Delta(\boldsymbol{r})$ nichts. Der Landau-Diamagnetismus und andere Eigenschaften, die der Normalleiter im Magnetfeld besitzt, bleiben unberücksichtigt. $M(0, \boldsymbol{A})$ bezieht sich auf die gleiche Fermi-Energie $\zeta$ wie $M(\Delta, \boldsymbol{A})$, weswegen die mittleren Teilchendichten verschieden sein können. Der Unterschied ist allerdings wegen $\Delta / \hbar \omega, \hbar \omega / \zeta \ll 1$ vernachlässigbar. Daher hebt sich der erste Summand in Gl. (A 10) gegen einen entsprechenden aus $\mathrm{M}(0, \boldsymbol{A})$.

Zur Umordnung der rechten Seite von Gl. (A 8) benutzen wir die Formel

$$
\sum_{\boldsymbol{k}} f\left((\boldsymbol{k}-\boldsymbol{q})^{2}, \boldsymbol{k}^{2}\right) \boldsymbol{k}_{i} \boldsymbol{k}_{l}=\frac{1}{2} \sum_{\boldsymbol{k}} f\left((\boldsymbol{k}-\boldsymbol{q})^{2}, \boldsymbol{k}^{2}\right) \boldsymbol{k}^{2}\left\{\left(1-\frac{(\boldsymbol{k} \boldsymbol{q})^{2}}{\boldsymbol{k}^{2} \boldsymbol{q}^{2}}\right) \delta_{i l}-\left(1-3 \frac{(\boldsymbol{k} \boldsymbol{q})^{2}}{\boldsymbol{k}^{2} \boldsymbol{q}^{2}}\right) \frac{\boldsymbol{q}_{i} \boldsymbol{q}_{l}}{\boldsymbol{q}^{2}}\right\}(i, l=x, y, z)
$$

$f$ ist eine beliebige Funktion von zwei Variablen. Damit ergibt sich $M_{1}(\Delta, \boldsymbol{A})-M_{1}(0, \boldsymbol{A})=$

$$
\begin{aligned}
&-\frac{e^{2}}{2 m c^{2}} \sum_{\boldsymbol{k q}}\left[\frac{\hbar^{2} \boldsymbol{k}^{2}}{2 m}\left(1-\frac{(\boldsymbol{k} \boldsymbol{q})^{2}}{\boldsymbol{k}^{2} \boldsymbol{q}^{2}}\right) L\left(\varepsilon, \varepsilon^{\prime}\right)-\frac{2 m\left|\Delta_{0}\right|^{2}}{\hbar^{2} \boldsymbol{q}^{2}} \frac{\varepsilon-\varepsilon^{\prime}}{\varepsilon+\varepsilon^{\prime}}\left(\frac{1}{E} \tanh \left(\frac{1}{2} \beta E\right)\right.\right.\left.\left.-\frac{1}{E^{\prime}} \tanh \left(\frac{1}{2} \beta E^{\prime}\right)\right)\right] \\
&-\frac{1}{2}\left(\frac{e}{\hbar c}\right)^{2} \sum_{\boldsymbol{k} \boldsymbol{q}} \frac{\varepsilon-\varepsilon^{\prime}}{\boldsymbol{q}^{4}}\left[\left(\frac{\varepsilon}{E} \tanh \left(\frac{1}{2} \beta E\right)-\tanh \left(\frac{1}{2} \beta \varepsilon\right)\right)-\left(\frac{\varepsilon^{\prime}}{E^{\prime}} \tanh \left(\frac{1}{2} \beta E_{i l}-\frac{\boldsymbol{q}_{i} \boldsymbol{q}_{l}}{\boldsymbol{q}^{2}}\right)-\tanh \left(\frac{1}{2} \beta \varepsilon^{\prime}\right)\right)\right]|(\boldsymbol{q} \boldsymbol{A}(\boldsymbol{q}))|^{2}
\end{aligned}
$$$$
-\left(\frac{e}{\hbar c}\right)^{2} \sum_{\boldsymbol{k q}} \frac{1}{\boldsymbol{q}^{2}} \frac{\varepsilon-\varepsilon^{\prime}}{\varepsilon+\varepsilon^{\prime}}\left[\frac{1}{E} \tanh \left(\frac{1}{2} \beta E\right)-\frac{1}{E^{\prime}} \tanh \left(\frac{1}{2} \beta E^{\prime}\right)\right]\left|\Delta_{0}\right|^{2}\left(\boldsymbol{A}(\boldsymbol{q}) \boldsymbol{A}^{*}(\boldsymbol{q})\right) \text {, }
$$ 


$$
\begin{aligned}
& L\left(\varepsilon, \varepsilon^{\prime}\right)=\frac{1}{\varepsilon^{2}-\varepsilon^{\prime 2}}\left\{\left[\left(E+\frac{\varepsilon \varepsilon^{\prime}+\left|\Delta_{0}\right|^{2}}{E}\right) \tanh \left(\frac{1}{2} \beta E\right)-\left(E^{\prime}+\frac{\varepsilon \varepsilon^{\prime}+\left|\Delta_{0}\right|^{2}}{E^{\prime}}\right) \tanh \left(\frac{1}{2} \beta E^{\prime}\right)\right]\right. \\
&\left.-\left(\varepsilon+\varepsilon^{\prime}\right)\left[\tanh \left(\frac{1}{2} \beta \varepsilon\right)-\tanh \left(\frac{1}{2} \beta \varepsilon^{\prime}\right)\right]\right\} .
\end{aligned}
$$

Die zweite Zeile der rechten Seite von Gl. (A 17) liefert einen mit $\hbar \omega / \zeta$ verschwindenden Beitrag. Der Ausdruck in der ersten Zeile ist eichinvariant. Die dritte Zeile soll mit $M_{2}$ und $M_{3}$ so kombiniert werden, daß die Phase von $\Delta(\boldsymbol{r})$ ein Eichpotential in $\boldsymbol{A}(\boldsymbol{r})$ kompensiert. $M_{2}$ kann mit Gl. (A 16) in der Form

$$
M_{2}=+\frac{e}{2 \hbar c} \sum_{k q} \frac{1}{\boldsymbol{q}^{2}} \frac{\varepsilon-\varepsilon^{\prime}}{\varepsilon+\varepsilon^{\prime}}\left[\frac{1}{E} \tanh \left(\frac{1}{2} \beta E\right)-\frac{1}{E^{\prime}} \tanh \left(\frac{1}{2} \beta E^{\prime}\right)\right]\left(\Delta_{0}^{*} \Delta(\boldsymbol{q})\left(\boldsymbol{q} \boldsymbol{A}^{*}(\boldsymbol{q})\right)+\text { c.c. }\right)
$$

und $M_{3}$ in der Form

$$
\begin{aligned}
M_{3}= & -\frac{1}{2} \sum_{k \boldsymbol{q}} \frac{1}{E} \tanh \left(\frac{1}{2} \beta E\right)\left|\Delta(\boldsymbol{q})-\Delta_{0} \delta_{\boldsymbol{q}, 0}\right|^{2}-\frac{1}{2} \sum_{k \boldsymbol{q}} \frac{\left|\Delta(\boldsymbol{q})-\Delta_{0} \delta_{\boldsymbol{q}, 0}\right|^{2}}{\varepsilon^{2}-\varepsilon^{\prime 2}}\left|\Delta_{0}\right|^{2}\left(\frac{1}{E} \tanh \left(\frac{1}{2} \beta E\right)\right. \\
& \left.-\frac{1}{E^{\prime}} \tanh \left(\frac{1}{2} \beta E^{\prime}\right)\right)-\frac{1}{4} \sum_{k \boldsymbol{q}} \frac{\varepsilon-\varepsilon^{\prime}}{\varepsilon+\varepsilon^{\prime}}\left(\frac{1}{E} \tanh \left(\frac{1}{2} \beta E\right)-\frac{1}{E^{\prime}} \tanh \left(\frac{1}{2} \beta E^{\prime}\right)\right)\left|\Delta(\boldsymbol{q})-\Delta_{0} \delta_{\boldsymbol{q}, 0}\right|^{2}
\end{aligned}
$$

geschrieben werden, so daß wir

$$
\begin{aligned}
& M(\Delta, \boldsymbol{A})-M(0, \boldsymbol{A})=\frac{1}{V_{0}} \int_{\Omega} \mathrm{d}^{3} \boldsymbol{r}|\Delta(\boldsymbol{r})|^{2}+\int_{\Omega} \mathrm{d}^{3} \boldsymbol{r} m\left(\left|\Delta_{0}\right|^{2}\right)-\underset{2}{2} \sum_{\substack{k \boldsymbol{q} \\
\mid<\hbar \omega}} \frac{1}{E} \tanh \left(\frac{1}{2} \beta E\right)\left|\Delta(\boldsymbol{q})-\Delta_{0} \delta_{\boldsymbol{q}, 0}\right|^{2} \\
& +\frac{1}{8} \sum_{k \boldsymbol{q}}\left[\frac{1}{E^{3}} \tanh \left(\frac{1}{2} \beta E\right)-\frac{\beta}{2 E^{2}}\left(\cosh \left(\frac{1}{2} \beta E\right)\right)^{-2}\right]\left|\left(\Delta(\boldsymbol{q})-\Delta_{0} \delta_{q, 0}\right) \Delta_{0}{ }^{*}+\Delta_{0}\left(\Delta^{*}(-\boldsymbol{q})-\Delta_{0}{ }^{*} \delta_{q, 0}\right)\right|^{2} \\
& +\frac{\Omega}{2} \sum_{\boldsymbol{q}} K_{3}(\boldsymbol{q})\left|\left(\Delta(\boldsymbol{q})-\Delta_{0} \delta_{\boldsymbol{q}, 0}\right) \Delta_{0}{ }^{*}+\Delta_{0}\left(\Delta^{*}(-\boldsymbol{q})-\Delta_{0}^{*} \delta_{\boldsymbol{q}, 0}\right)\right|^{2} \\
& +\frac{\Omega}{2 m} \sum_{\boldsymbol{q}} K_{1}(\boldsymbol{q})\left[(\hbar \boldsymbol{q})^{2}\left|\Delta(\boldsymbol{q})-\Delta_{0} \delta_{q, 0}\right|^{2}-\frac{2 e \hbar}{c}\left(\Delta_{0}^{*} \Delta(\boldsymbol{q})\left(\boldsymbol{q} \boldsymbol{A}^{*}(\boldsymbol{q})\right)+\text { c.c }\right)+\frac{4 e^{2}}{c^{2}}\left|\Delta_{0}\right|^{2}\left(\boldsymbol{A}(\boldsymbol{q}) \boldsymbol{A}^{*}(\boldsymbol{q})\right)\right] \\
& -\frac{2 e^{2} \Omega}{m c^{2}} \sum_{\boldsymbol{q}} K_{2}(\boldsymbol{q}) \boldsymbol{A}_{i}(\boldsymbol{q})\left(\boldsymbol{q}^{2} \delta_{i l}-\boldsymbol{q}_{i} \boldsymbol{q}_{l}\right) \boldsymbol{A}_{l}^{*}(\boldsymbol{q}) \\
& \text { mit } \quad m\left(\left|\Delta_{0}\right|^{2}\right)=\frac{1}{\Omega} \sum_{k}\left(\varepsilon-E-2 k T \log \frac{1+\exp \{-\beta E\}}{1+\exp \{-\beta \varepsilon\}}\right)
\end{aligned}
$$

erhalten. Dabei ist

$$
\begin{gathered}
K_{1}(\boldsymbol{q})=-\frac{1}{\Omega} \sum_{k} \frac{m}{2 \hbar^{2} \boldsymbol{q}^{2}} \frac{\varepsilon-\varepsilon^{\prime}}{\varepsilon+\varepsilon^{\prime}}\left[\frac{1}{E} \tanh \left(\frac{1}{2} \beta E\right)-\frac{1}{E^{\prime}} \tanh \left(\frac{1}{2} \beta E^{\prime}\right)\right], \\
K_{2}(\boldsymbol{q})=\frac{\left|\Delta_{0}\right|^{2}}{\boldsymbol{q}^{2}} K_{1}(\boldsymbol{q})+\frac{1}{\Omega} \sum_{k} \frac{\hbar^{2} \boldsymbol{k}^{2}}{8 m \boldsymbol{q}^{2}}\left(1-\frac{(\boldsymbol{k} \boldsymbol{q})^{2}}{\boldsymbol{k}^{2} \boldsymbol{q}^{2}}\right) L\left(\varepsilon, \varepsilon^{\prime}\right), \\
K_{3}(\boldsymbol{q})=-\frac{1}{2 \Omega} \sum_{\boldsymbol{k}}\left\{\frac{1}{\varepsilon^{2}-\varepsilon^{\prime 2}}\left[\frac{1}{E} \tanh \left(\frac{1}{2} \beta E\right)-\frac{1}{E^{\prime}} \tanh \left(\frac{1}{2} \beta E^{\prime}\right)\right]+\frac{1}{2 E^{2}}\left[\frac{1}{E} \tanh \left(\frac{1}{2} \beta E\right)\right.\right. \\
\left.\left.-\frac{\beta}{2}\left(\cosh \left(\frac{1}{2} \beta E\right)\right)^{-2}\right]\right\} .
\end{gathered}
$$

Die Rücktransformation von Gl. (A 21) in den Ortsraum liefert Gl. (27). Die Raumwinkelintegration über $\boldsymbol{k}$ in $K_{1}(\boldsymbol{q}), K_{2}(\boldsymbol{q})$ und $K_{3}(\boldsymbol{q})$ läßt sich durchführen, und man erhält die in den Gln. $(29 \mathrm{a}, \mathrm{b}, \mathrm{c})$ angegebene Darstellung. 